Undergraduate Students - Population, Health \& Wellness

\title{
Unraveling Gene Expression Profiles of Cardiac Genes That Participate in Embryonic Development of Congenital Heart Defects Using Chick Embryo Amal Nasr ${ }^{1}$, Aya Omar ${ }^{1}$, Maha Alser 2 , Fatiha Benslimane 2 , Hüseyin Yakçın² ${ }^{1}$ Biomedical Science Department, College of Health Sciences, Qatar University, Doha, QATAR
}

${ }^{2}$ Biomedical Research Center, Qatar University, Doha, QATAR

\section{ABSTRACT}

Hypoplastic left heart syndrome (HLHS) is a rare but serious subtype of congenital heart defects (CHDs) at which the hemodynamics are disturbed. In this project, HLHS was introduced surgically by left atrial ligation (LAL) to embryonic chicks and the subsequent effects of it were studied. Different tests were done post-LAL to study cardiac morphology,

function, and gene expression of cardiac Figure 1: a heart with hypoplastic markers.

\section{INTRODUCTION}

- Congenital heart defects (CHDs) are heart disorders that may arise when cardiogenesis is disturbed. CHDs affects $1-1.2 \%$ of newborns and considered as the primary cause of death in children under the age of one year.

Hypoplastic left heart syndrome (HLHS) is a severe subtype of CHDs where the left ventricle volume is remarkably reduced Thus, the heart will not be able to support the systemic circulation.

The etiology of most CHDs cases believed to be because of disturbed hemodynamics.

- LAL is a surgical intervention that induce HLHS in vivo.

Chicken embryos are commonly used animal models as it resembles the configuration of the four chambers and the four valves of human.

\section{METHODOLOGY}

\section{Chick embryo culture}

Fertilized eggs were incubated under $37.5^{\circ} \mathrm{C}, 60 \%$ humidity, and continuous rocking, the eggs were opened at ED3.

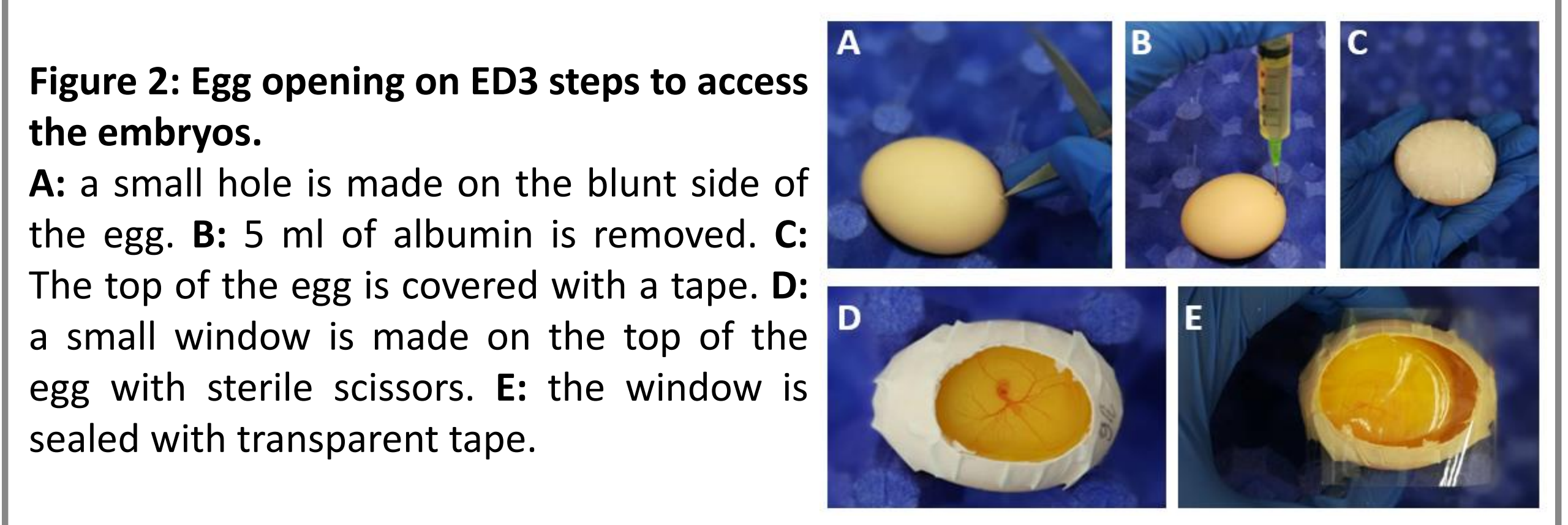

Introducing HLHS via Left Atrial Ligation (LAL) Figure 3: LAL steps on ED 4: A.

Opening embryonic chorionic

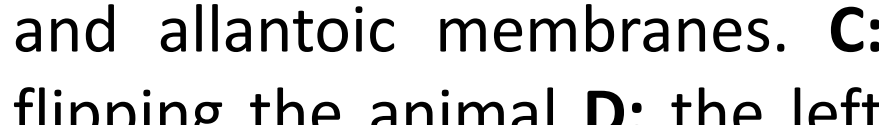

side of the embryo. E: opening

the pericardium $F$ : placing the
$10-0$ knot on the top of the atrium. G: the an
tightening the knot.

Heart function assessment via echocardiography

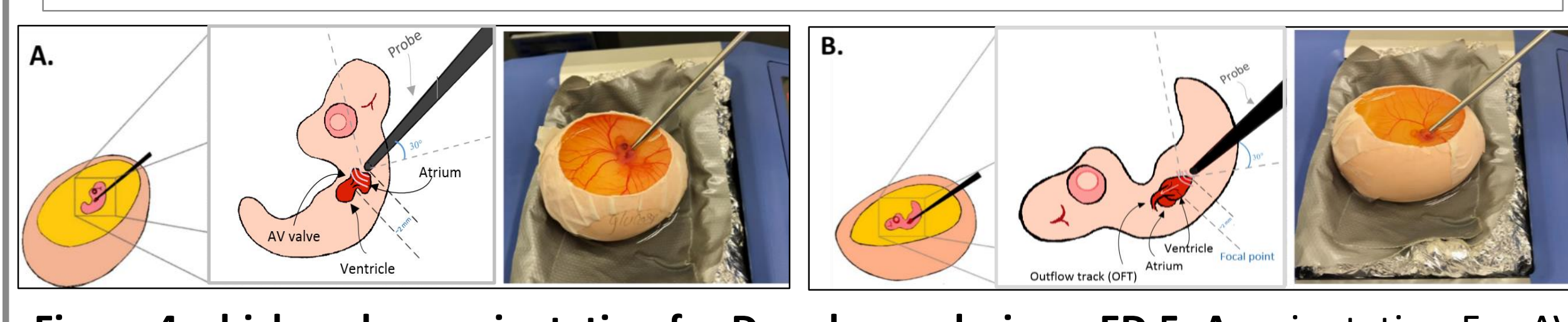

Figure 4: chick embryo orientation for Doppler analysis on ED 5. A. orientation For AV valve blood flow velocity measurement. B. orientation For OFT blood flow velocity

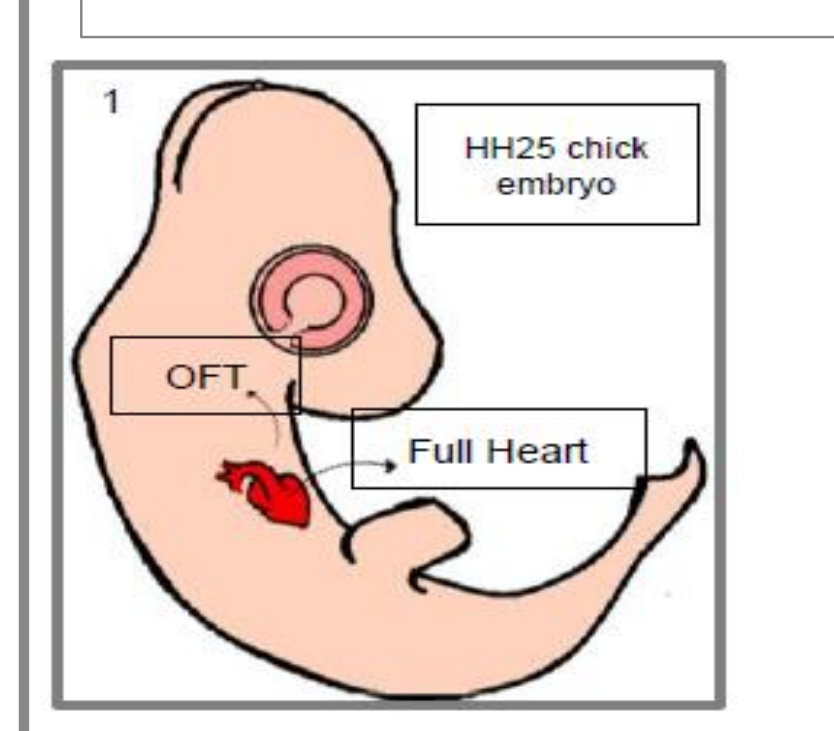

Full heart isolation
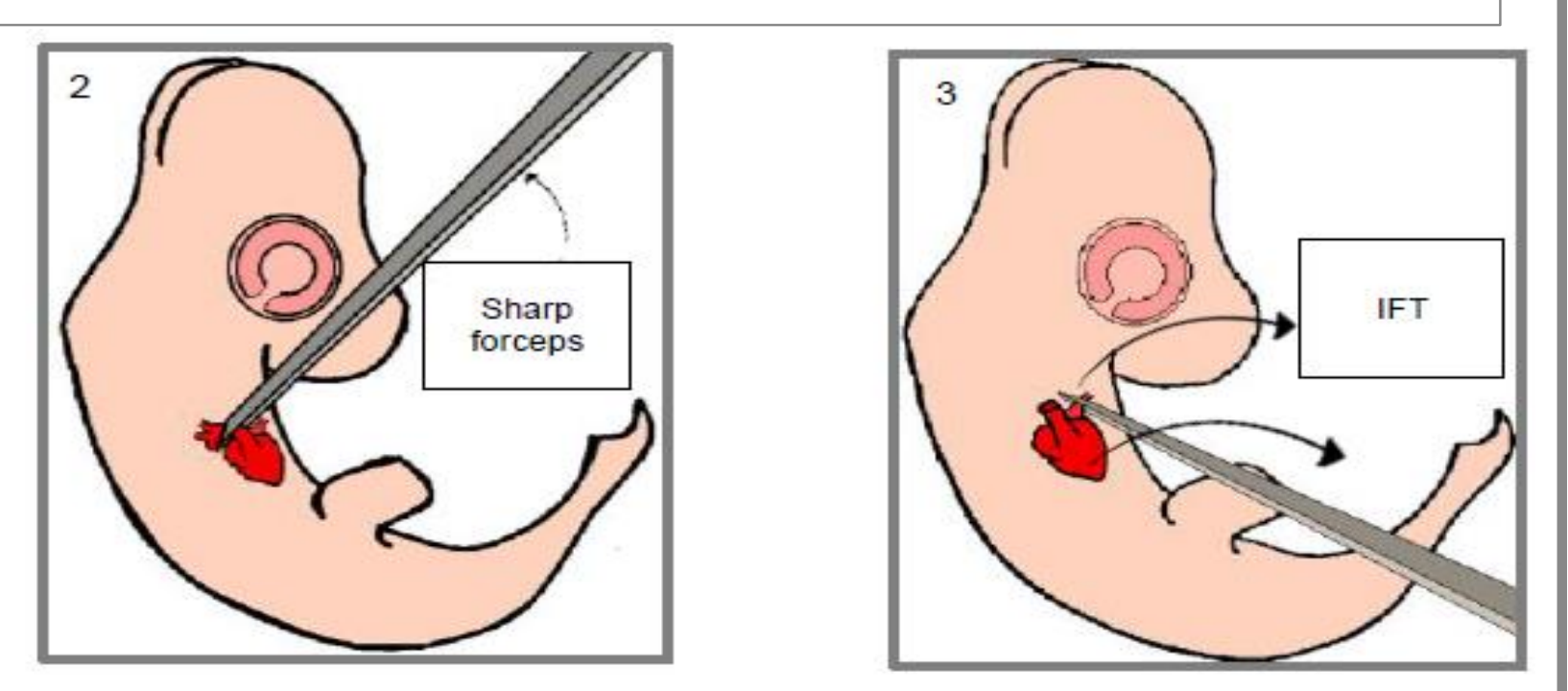

Figure 5: Full heart extraction steps on ED 5, 24-hr post-LAL: 1. The embryo is taken, OFT and IFT are located. 2. Using sharp forceps the OFT is cut. 3. The heart is flipped $4 \%$ PFA to be fixed for histology.

Relative gene expression of cardiac markers RNA isolation and estimation, cDNA synthesis, RT-PCR

\section{Histology}

- Paraffin-embedded tissue sectioning

- Hematoxylin-Eosin (H\&E) staining

\section{RESULTS}

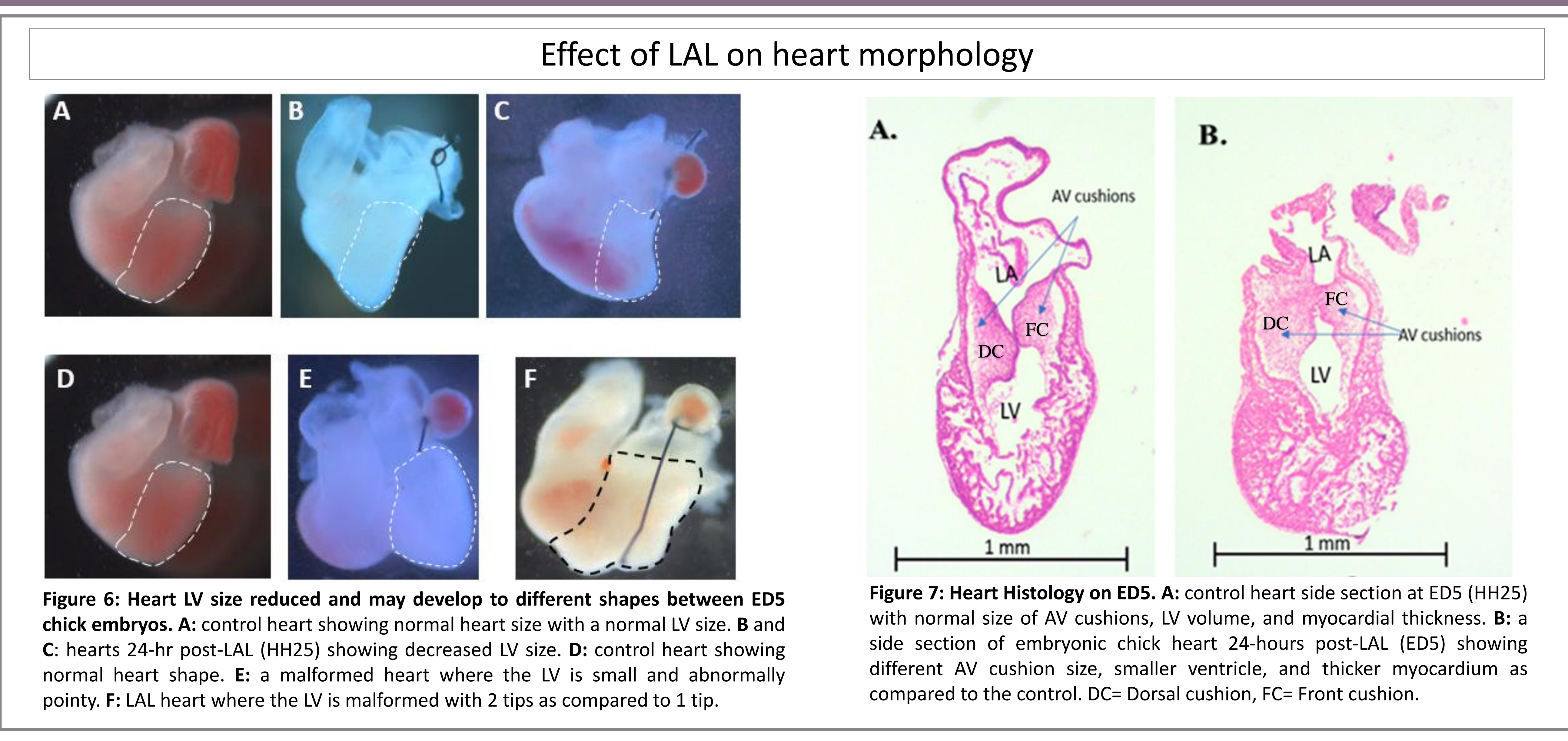

Effect of LAL on heart function

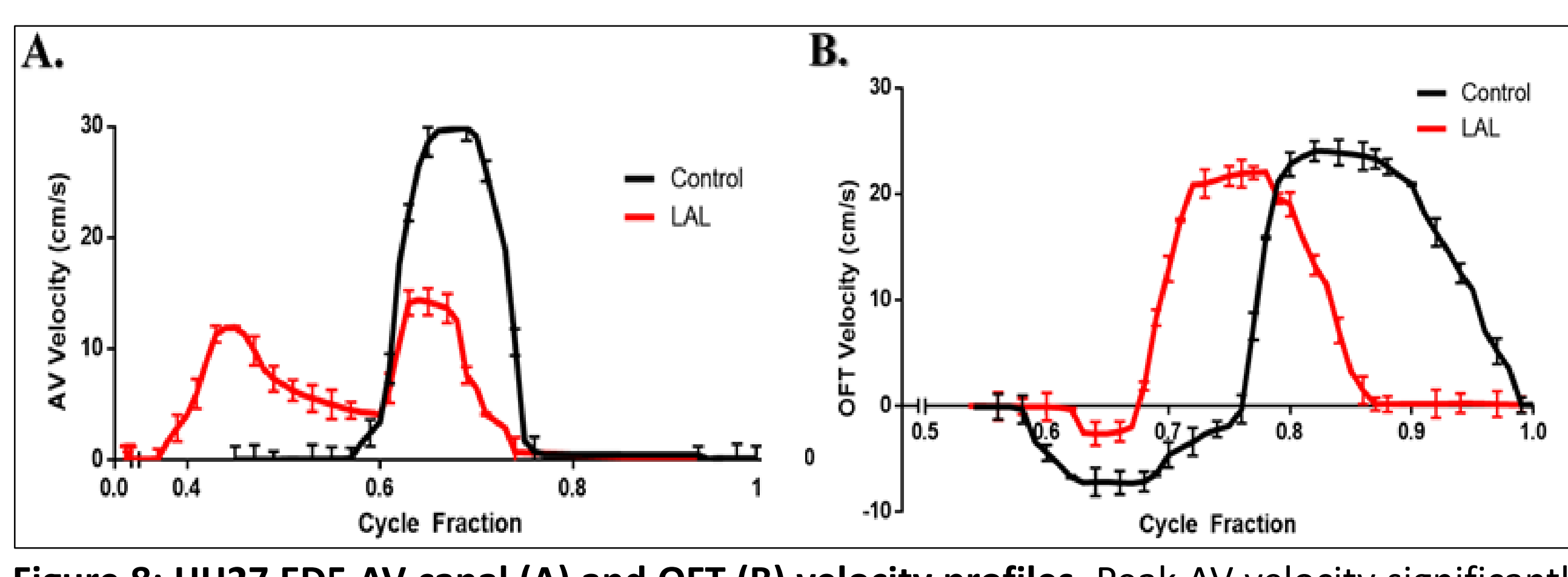

Figure 8: HH27 ED5 AV canal (A) and OFT (B) velocity profiles. Peak AV velocity significantly decreased for LAL heart. No significant change in OFT peak velocity. Duration time in AV valve significantly increased. In the OFT signal, the whole profile changed after LAL, the wave started earlier than control,
OFT signal disappeared in the LAL.
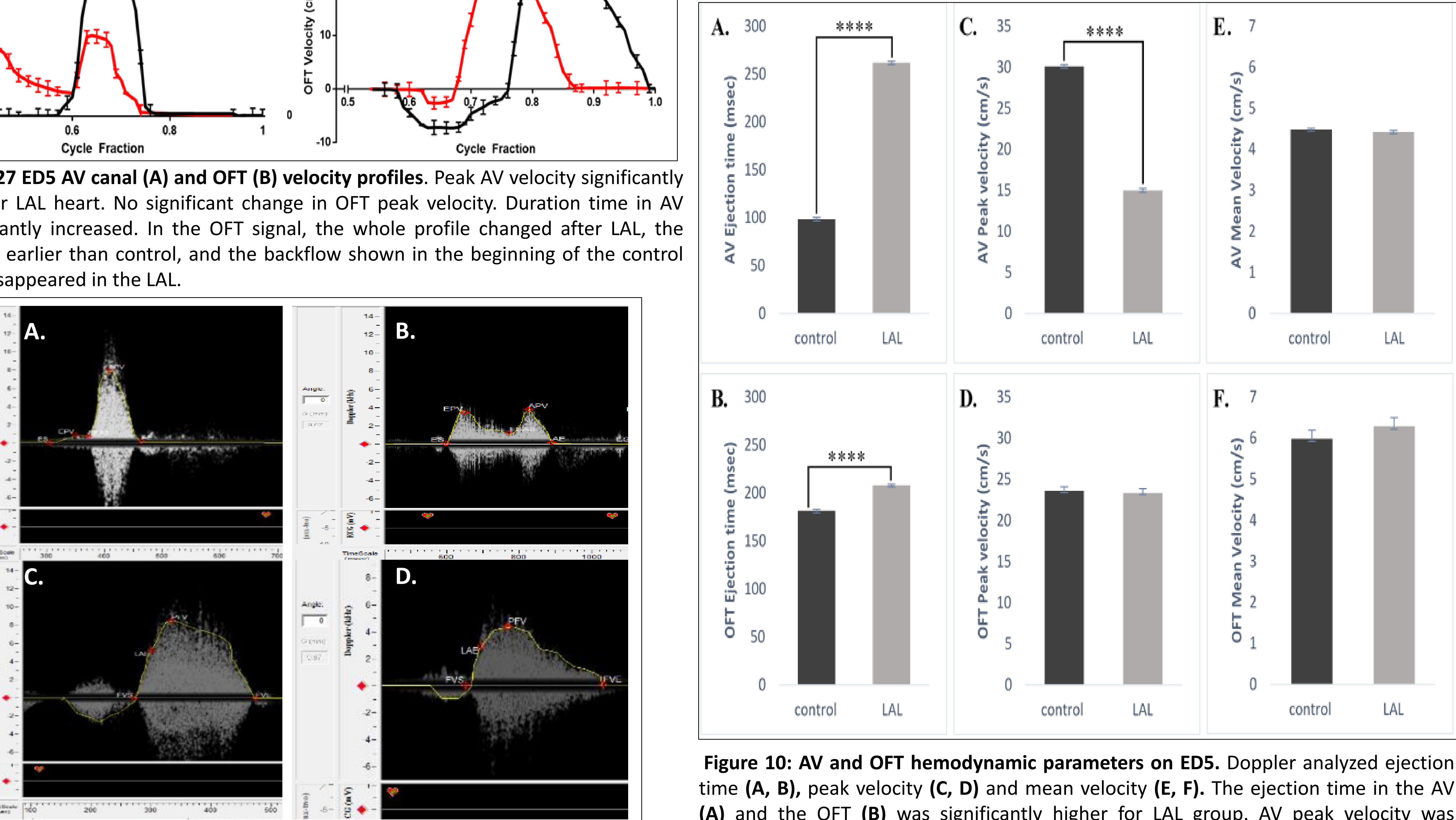

Figure 10: AV and OFT hemodynamic parameters on ED5. Doppler analyzed ejection ime (A, B), peak velocity (C, D) and mean velocity (E, F). The ejection time in the AV (A) and the OFT (B) was significantly higher for LAL group. AV peak velocity was Figure 9: Measurements of Doppler Signal Processing Work Station Software on ED5. significantly lower in the AV (C), but no significant change in OFT (D) of the LAL group. \begin{tabular}{l}
$\begin{array}{l}\text { Hemodynamics were analyzed to detect AV signal in } \mathbf{A} \text {. control and B. LAL and detect the } \\
\text { OFT signal in C. control and D. LAL. } \\
\text { group and } 12 \text { for LAL group. The significant marks reflect the standard error. }\end{array}$ \\
\hline
\end{tabular}

Effect of LAL on cardiac markers gene expiration

LAL group has alterations in the expression level of all cardiac markers as compared to normal chick embryo hearts. LAL has significantly decreased the expression level of VEGF-a, TGF-b, BMP2, NKX2.5, and KLF2. No significant effect was shown of ACTA2.

Figure 11: Relative gene expression level fold changes of different genes from LAL HH25 full hearts RNA extracts using RT-PCR. LAL led to significantly lowered level of most cardiac markers and shear stress markers. The expression was normalized to $18 \mathrm{~S}$
ANOVA, and the data is presented as mean fold chane ${ }^{*}=$ significant effect $(p<0.05),{ }^{* *}=$ highly significant effect $(p<0.01)$.

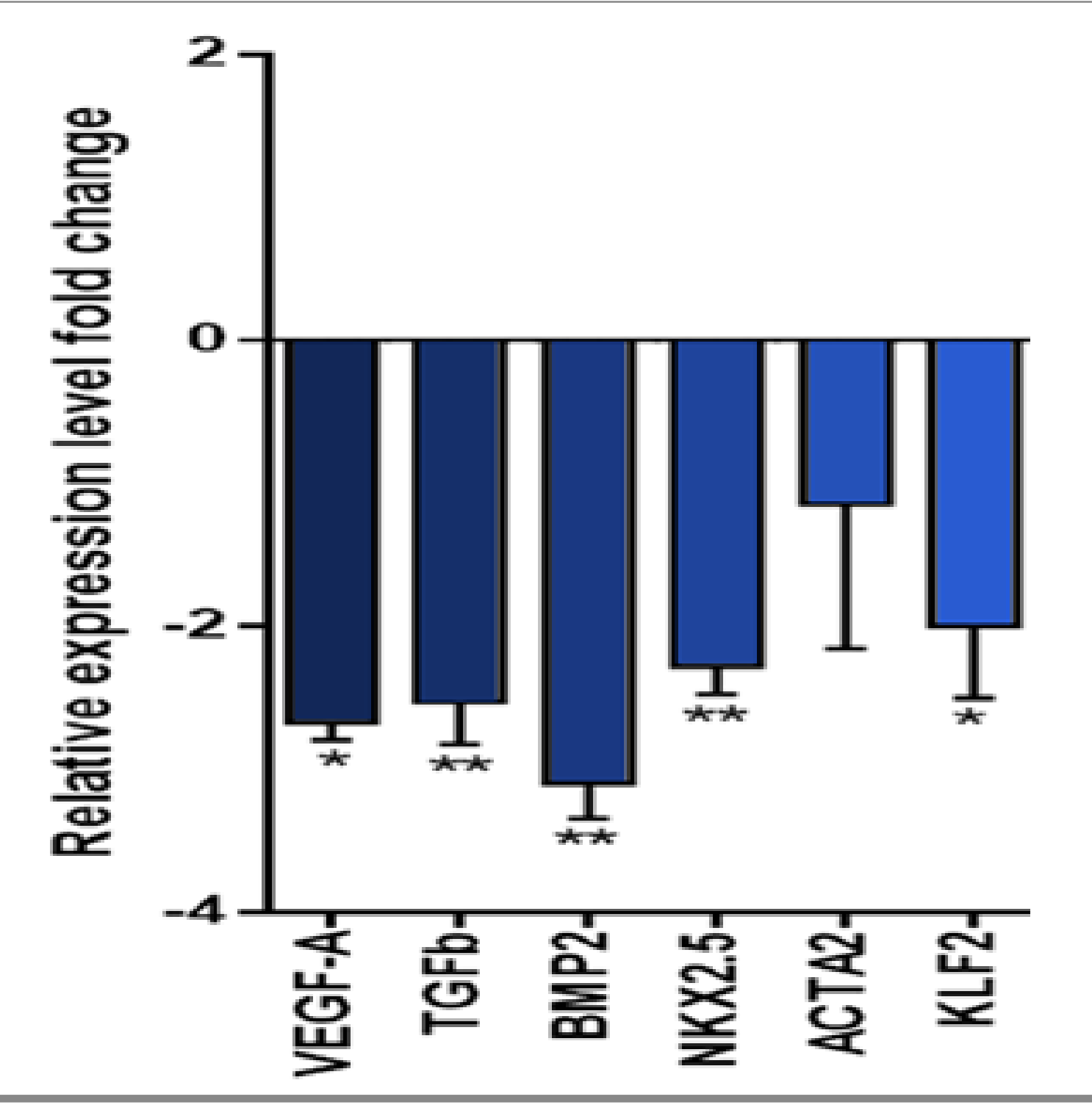

\section{CONCLUSION}

Disturbing hemodynamics can lead to drastic malformations in the embryonic heart morphology. - Disturbed hemodynamics negatively affect cardiac parameters and alter the heart ability to function.

Changing hemodynamics leads to significant decrease in cardiac development markers expression level

- These findings put the starting to understand HLHS as a disease and think of candidate therapeutic techniques.

\section{REFERENCES}

Lindsey, S. E., Butcher, J. T., \& Yalcin, H. C. (2014). Mechanical regulation of cardiac development. Frontiers in Physiology, 5.

Tobita, K., \& Keller, B. B. (2000). Right and left ventricular wall deformation patterns in normal and left heart hypoplasia chick embryos. American Journal of Physiology-Heart and Circulatory Physiology, 279(3).

\section{ACKNOWLEDGMENTS}

Our deepest gratitude to our graduation project supervisor Huseyin Yalcin and project coordinator Maha Alser. Specia thanks to the BRC, LARC and HCY lab team. 